\title{
Exploring Student Check-In Behavior for Improved Point-of-Interest Prediction
}

\author{
Mengyue Hang \\ Computer Science Department \\ Purdue University \\ hangm@purdue.edu
}

\author{
Ian Pytlarz \\ Office of Institutional Research \\ Purdue University \\ ipytlarz@purdue.edu
}

\author{
Jennifer Neville \\ Computer Science and Statistics \\ Departments \\ Purdue University \\ neville@purdue.edu
}

\begin{abstract}
With the availability of vast amounts of user visitation history on location-based social networks (LBSN), the problem of Point-ofInterest (POI) prediction has been extensively studied. However, much of the research has been conducted solely on voluntary checkin datasets collected from social apps such as Foursquare or Yelp. While these data contain rich information about recreational activities (e.g., restaurants, nightlife, and entertainment), information about more prosaic aspects of people's lives is sparse. This not only limits our understanding of users' daily routines, but more importantly the modeling assumptions developed based on characteristics of recreation-based data may not be suitable for richer check-in data. In this work, we present an analysis of education "check-in" data using WiFi access logs collected at Purdue University. We propose a heterogeneous graph-based method to encode the correlations between users, POIs, and activities, and then jointly learn embeddings for the vertices. We evaluate our method compared to previous state-of-the-art POI prediction methods, and show that the assumptions made by previous methods significantly degrade performance on our data with dense(r) activity signals. We also show how our learned embeddings could be used to identify similar students (e.g., for friend suggestions).
\end{abstract}

\section{KEYWORDS}

Location-based social networks, network embedding, heterogeneous graphs, representation learning.

\section{ACM Reference Format:}

Mengyue Hang, Ian Pytlarz, and Jennifer Neville. 2018. Exploring Student Check-In Behavior for Improved Point-of-Interest Prediction. In KDD 2018: 24th ACM SIGKDD International Conference on Knowledge Discovery \& Data Mining, August 19-23, 2018, London, United Kingdom. ACM, New York, NY, USA, 10 pages. https://doi.org/10.1145/3219819.3219902

\section{INTRODUCTION}

Millions of check-in records in location-based social networks (LB$\mathrm{SNs}$ ) provide an opportunity to study users' mobility pattern and social behavior from a spatial-temporal perspective. In recent years,

Permission to make digital or hard copies of all or part of this work for personal or classroom use is granted without fee provided that copies are not made or distributed for profit or commercial advantage and that copies bear this notice and the full citation on the first page. Copyrights for components of this work owned by others than ACM must be honored. Abstracting with credit is permitted. To copy otherwise, or republish, to post on servers or to redistribute to lists, requires prior specific permission and/or a fee. Request permissions from permissions@acm.org.

KDD 2018, August 19-23, 2018, London, United Kingdom

(C) 2018 Association for Computing Machinery.

ACM ISBN 978-1-4503-5552-0/18/08 . \$ \$15.00

https://doi.org/10.1145/3219819.3219902 the point-of-interest (POI) recommendation/prediction problem has attracted significant attention [19], [20], [5], [21], [17], particularly for advertising and personalization. In POI tasks, the goal is to use user behavioral data to model users' activities at different locations and times, and then make predictions (or recommendations) for relevant venues based on their current context (including spatial, temporal, and other contextual information).

While POI predictions have broad applicability to myriad organizations, to date research has focused on developing POI methods based solely on voluntary check-in datasets collected from online social network apps such as Foursquare or Yelp [10], [6]. While these data contain rich information about recreational activities (e.g., restaurants, nightlife, and entertainment), the reliance on voluntary reporting results in sparse information about more prosaic aspects of daily life (e.g., offices, errands, houses). Moreover, recreation-based check-in data may bias conclusions drawn about mobility patterns or personal preferences. For example, Foursquare users often visit a POI only once, so the users' check-ins may not be sufficient to derive preferences for venues themselves, but only for venue categories. Also since check-ins to location-based social networks are often sporadic [10], it can be difficult to identify consistent user patterns.

In this work, we present the first analysis of a spatio-temporal educational "check-in" dataset, with the aim of using POI predictions to personalize student recommendations (e.g., clubs, friends, study locations) and to understand behavior patterns that increase student retention and satisfaction. The results also provide a better idea of how campus facilities are utilized and how students connect with each other. The Purdue University "check-in" data records (anonymized) users' access to WiFi access points on campus, with venue information about locations (e.g., dining hall, library, dorm, gym). Specifically, we analyze WiFi access history across on-campus buildings, for all freshmen over one semester.

Compared to well-known check-in datasets like Foursquare, these data contain (1) more active users, (2) a richer set of daily activities (e.g., study, dine, exercise, rest), and (3) well-annotated spatial range (i.e., on campus). These characteristics make it easier to analyze the unique properties of user check-in data and extract interesting social and mobility patterns. Notably the WiFi access logs provide better temporal resolution than previous LBSN datasets, since a user "checks-in" whenever her device sends or receives a packet through a wireless connection. Similar data are collected by GPS trackers, where location observations are passively recorded [23]. But while GPS tracking provides more extensive information about users' movements, it does not provide the rich venue and activity information associated with check-in data. 
POI prediction and recommendation tasks are different from more traditional recommendation tasks because they involve a more structured, context-rich environment [13]. In addition to userPOI check-in frequencies, the users and POIs are usually associated with a rich set of attributes, such as POI category, spatio-temporal information, personal activity. A heterogeneous graph structure is thus a natural choice to for spatio-temporal POI prediction tasks, since it is more amenable to representing and reasoning with rich context compared to tensor factorization methods (e.g., [5]).

Recently, methods which learn graph representations by embedding nodes in a vector space have gained traction from the research community, and graph embedding methods have been widely adopted for a variety of tasks, including text mining [14], online event detection [22] and author identification [2]. In this work, we extend these efforts and propose a network-based embedding method called Embedding for Dense Heterogeneous Graphs (EDHG). Our approach (i) incorporates personal preferences, temporal patterns, and activity types into a sparse(r) view of the heterogeneous graph, (ii) uses global knowledge of the graph to generate negative samples, (iii) jointly learns vector representations for the nodes in the graph, i.e., users, POIs, time-slots, and then (iv) uses the learned representations for user and time specific POI recommendation.

We empirically evaluate the effectiveness of EDHG using POI prediction and friend suggestion tasks and show that it outperforms previous state-of-the-art POI recommendation methods. Our investigation shows that reason for the improvement stems from the process of (i) heterogeneous graph construction, and (ii) negative sampling. We show that the processes used in previous methods are more suitable to OSN check-in data based on sparse voluntary reporting, than dense(r) check-in data based on location tracking.

To summarize, our work makes the following contributions:

(1) Presents the first educational "check-in" dataset and explores its unique mobility and social characteristics;

(2) Identifies the challenges for time-aware POI prediction in educational check-in data based on increased density due to location-based tracking (compared to previous voluntaryreport LSBN data);

(3) Proposes a novel heterogeneous information network-based model to encode the relations between users, POIs, and timeslots, and evaluates its efficacy for POI and user recommendation tasks.

\section{DATA CHARACTERISTICS}

In this section, we discuss the characteristics of the Purdue educational "check-in" dataset and showcase its unique aspects compared to previous check-in data.

\subsection{Data sample}

In this work, we use two sample datasets from the Purdue Office of Instituional Research: (i) WiFi log data and (ii) building location profiles ${ }^{1}$. We consider a sample of the data restricted to freshmen students in the 2016-17 academic year. The $376 \mathrm{~Gb} \mathrm{WiFi} \log$ file contains over 1 billion entries, each of which records a data communication between a campus WiFi access point and a personal device

\footnotetext{
${ }^{1}$ Collected and analyzed anonymously, with IRB approval
}

\begin{tabular}{|c|l|l|}
\hline Item & Number & Description \\
\hline Users & 6250 & Freshmen \\
\hline POIs & 221 & On-campus buildings \\
\hline POI category & 4 & $\begin{array}{l}\text { Academic, Residential, Admin- } \\
\text { istration, Auxiliary }\end{array}$ \\
\hline POI functionality & 7 & $\begin{array}{l}\text { Residence, Recreation, Dining, } \\
\text { Exercise, Library/Lab, Class- } \\
\text { rooms, Others }\end{array}$ \\
\hline Time span & $1 \mathrm{sem}$. & Fall: $08 / 22 / 16$ to $12 / 17 / 16$ \\
\hline
\end{tabular}

Table 1: Dataset description

in the time period 7/31/2016 to 6/30/2017. Each entry contains activity time (date, hour and minute), anonymized user id, MAC address, and building id. The building profile provides building information, including building id, name, category, and functionalities. Note that each building belongs to one category but might have multiple functionalities. We remove users with fewer than 100 check-ins. We also drop the check-in records generated by MAC addresses that only checkin at a single building, as these devices are likely to be stationary PCs in dorms or offices. Moreover, using class registration information, we attach a 'in-class' label for the record if the WiFi access point is in the building associated with their course schedule at that time/day. While, we retain these in-class checkins for the analysis in this section, we remove them for the modeling in Section 3, to focus the prediction task on less predictable user movements. The final processed sample has 540 million logs in total. More dataset statistics are shown in Table 1.

\subsection{Temporal dynamics of user preferences}

Figures 1a-1d show the students aggregated temporal preference for each type of activity in terms of the conditional probability $\operatorname{Pr}($ time $=\tau \mid$ activity $=a)$ for a given time slot $\tau$ and activity $a$ (e.g., Dining). We can see that different activities show unique temporal patterns. For example, on weekdays (Fig. 1a) students usually visit the dining halls (i.e., dining activity) around $12 \mathrm{pm}$ and $6 \mathrm{pm}$, and go to the gym around 8pm. Check-ins at the residence halls are visible throughout the day, reflecting the variability in students daily routines and the dorms' versatility.

Figures $1 \mathrm{a}$ and $1 \mathrm{~b}$ show the differences in time preferences for weekday and weekend, respectively. Students only have classes on Saturday morning, and they are more likely to start studying (including staying in the lab or library) at later times on weekends. For non-academic activities on weekends, visiting hours to the gym are more distributed owing to their more flexible schedule, and more students choose to have lunch rather than dinner on campus.

We also investigate if students' temporal preferences vary by major. Figures 1c and 1d, show the preferences for 302 computer science students and 267 Pharmacy students, respectively. We can see that the overall preferences are similar for Dining, while there are some differences in taking classes (shown in activity Class), staying in research labs/library (shown in activity Study), and exercise (shown in activity Gym). Specifically, Pharmacy students attend class more often from $11 \mathrm{am}$ to $12 \mathrm{pm}$, while CS students attend class from morning to afternoon. CS students spend more time in academic buildings (from 10am to $7 \mathrm{pm}$ ) than Pharmacy students who prefer to study in the morning and around noon. For 


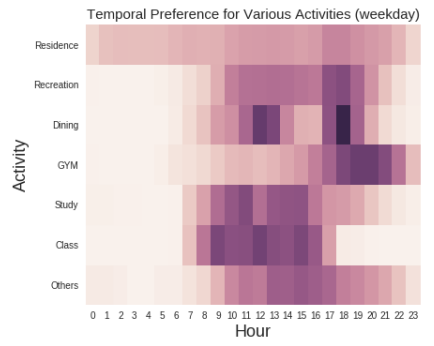

(a) Weekday

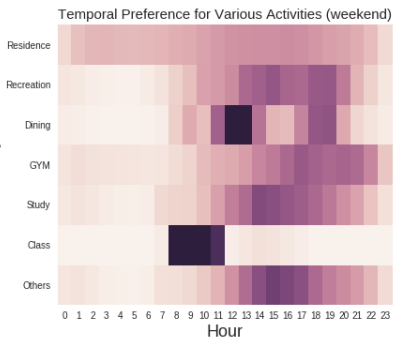

(b) Weekend

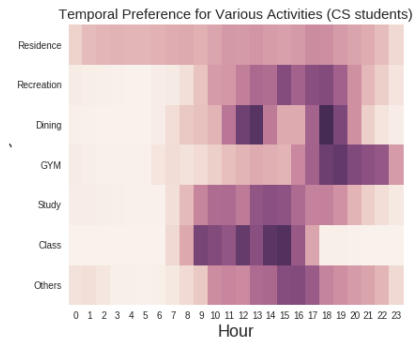

(c) CS

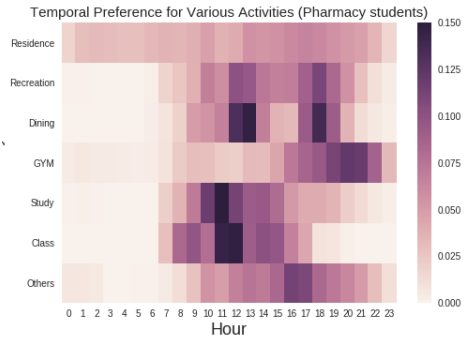

(d) Pharmacy

Figure 1: Hourly activity preference for (a) weekday, (b) weekend, (c) computer science students, and (d) pharmacy students

non-academic activities, students from both majors show similar temporal preference, while pharmacy students tend to go to the gym at later times.

\subsection{Co-visitation behavior}

While individual visitation histories can indicate temporal and spatial preference, in isolation they do not indicate relationships among the students. However, co-visitation events (i.e., when two students are in the same place at the same time), may be a noisy indicator of relations among students. Any one co-visitation event may be due to random chance, but a larger set of events, particularly when 'in-class' events are dropped, is likely to indicate student friendship. To the best of our knowledge, a study of user pairwise covisitation events hasn't been investigated in other spatio-temporal analyses.

Since our dataset contains discrete WiFi login records, we merge each user's consecutive logins in the same building, and assume that the user stays in the building throughout this period. For example, if a user checked in at the library every four to eight minutes from $5 \mathrm{pm}$ to $6 \mathrm{pm}$ with no checkins at other buildings in between, we will merge these check-ins and record that the user stayed in library from $5 \mathrm{pm}$ to $6 \mathrm{pm}$. In this way, we augment the visit history with duration time for each user, and use that to compute the pairwise co-visitation count matrix. As each co-visitation is per minute, the pairwise co-visitation count is the total time (in minutes) two users spend together in the same building. For example, the pair of users with the largest co-visitation count spent 38,129 minutes together, which is roughly 27 days, more than $25 \%$ of the semester. Note that the in-class check-ins are removed for computing co-visitation. In this way, we only consider the activity outside of class for analyzing co-visitation, which we believe is more informative for determining friend realtions. We will use the pairwise co-visitation count to examine the performance of our embeddings on a friend suggestion task in Section 4.5.

Once the visit history is augmented with duration time for each user, we compute the number of users visiting the same building at the same time. For each building, we calculate the number of unique visitors for each minute over the semester and filter out the moments when there are fewer than two visitors. We show the normalized histogram in Figure 2. It indicates that the number of users appearing at the same time in the building may reflect building categories, as co-visitation happens more frequently in dorms and the gym compared to academic building (e.g., CS).

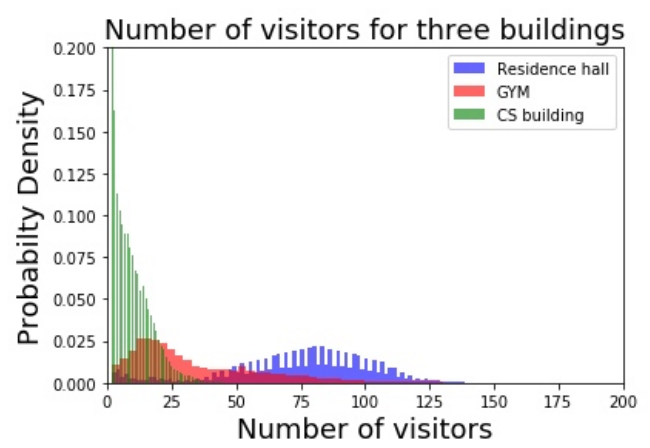

Figure 2: Histogram of co-visitation size for an academic building (CS), the Gym, and a residence hall, over all times.

\subsection{Exploration behavior}

We compare the exploration behaviors in our educational "check-in" dataset to traditional POI recommendation datasets like Foursquare and Gowalla. Figure 3 shows the average ratio of new POIs over all users for every new week. For example, the ratio at week two is the proportion of POIs visited during the second week that have not been visited in previously.

Compared with Foursquare users (Figure 3c) who keep exploring new POIs all year round, freshmen (Figure 3a) appear to explore the campus very quickly (within 2-3 weeks), and then stick to a fixed range of buildings over the remainder of the semester. But when we zoom into the first two weeks (Figure 3b), new students show similar exploration behaviors as in the Foursquare data, with 40 to 60 percent new POIs every day. This provides us with a unique opportunity to model two types of behaviors with different slices of the data: (1) the first few weeks of freshmen semester-exploring new places, and (2) the latter half of the semester-routinely visiting familiar places in a relatively limited activity range.

\section{PROPOSED EDHG METHOD}

In this section, we outline our proposed heterogeneous graph embedding method for POI prediction. Specifically, we consider a time-aware location prediction problem. Given a user and time slot (e.g., Monday $8 \mathrm{am}$ ), the model should predict a place that is most likely to be visited. 


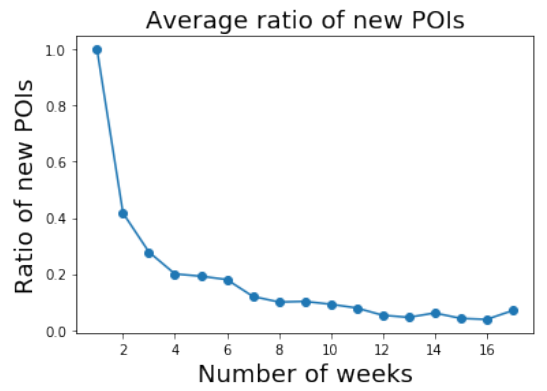

(a) Student "check-in" dataset

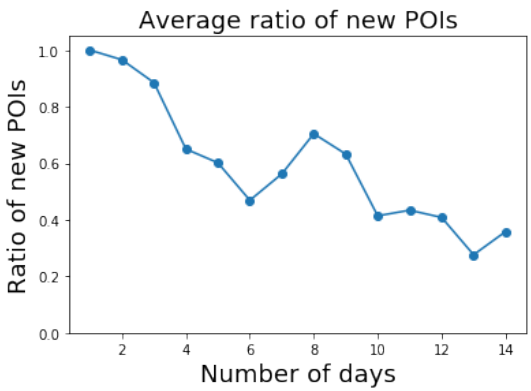

(b) Student "check-in" dataset

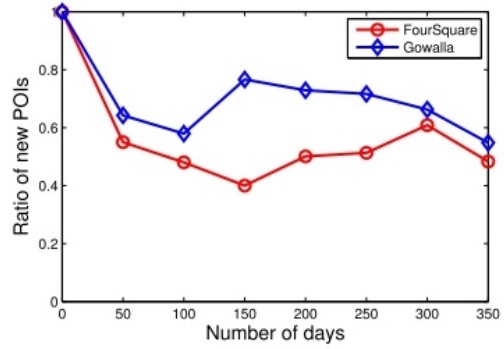

(c) Foursquare and Gowalla

Figure 3: Average ratio of new POIs: (a-b) Purdue data (weeks/days), and (c) foursquare and Gowalla dataset (from [4]).

We refer to our method as Embedding for Dense Heterogeneous Graphs (EDHG). It is designed specifically to reflect the characteristics of our educational check-in data, which is more dense than traditional LBSN check-in data. To better leverage contextual information, we propose a joint embedding model, which maps user, location, time and activity category into a common latent space.

In this section, we introduce EDHG step by step. We first construct a heterogeneous graph using the check-in records, then we learn continuous feature representations for vertices by capturing features of connectivity and structural similarity for pairs of nodes. In Sections 3.3-3.4, we discuss how to use the learned representations for POI prediction and friend suggestions, respectively.

\subsection{Heterogeneous Graph Construction}

Time indexing scheme. According to our data exploration results, the temporal characteristics of students behavior contain two aspects: (1) periodicity, and (2) preference variance. For example, students' check-ins have clear weekly cyclic patterns. Moreover, students usually visit academic buildings more on weekdays and stay at resident halls more on weekends.

In order to capture these temporal cyclic patterns, we designed a time indexing scheme to encode a standard time stamp to a particular time id. We consider the preference variance in two scales: hours of a day and different days of a week. First, a time stamp is divided into two slices in terms of weekday and hour slot. Next, we split a week into 7 days and a day into the following four sessions:

(1) Morning - hours between 6 am and 11:59 am

(2) Afternoon - hours between $12 \mathrm{pm}$ and 4:59 pm

(3) Evening - hours between $5 \mathrm{pm}$ and 11:59 pm

(4) Night - hours between 12 am and 5:59 am

This totals 28 distinct time slot ids, which can represent both weekly and daily preference variance.

Weighted graph construction. We construct a weighted heterogeneous information network by aggregating the check-in records and venue information. An example is shown in Figure 4 with eight check-in records. In this example, $u_{1}, u_{2}$ denote two users, $b_{1}, b_{2}, b_{3}$ denote three buildings/POIs, $t_{1}, t_{2}, t_{3}$ denote three time slots, and $a_{1}, a_{2}, a_{3}$ are three types of activities corresponding to POI functionalities, which we obtained from the venue information. Our model considers three types of edges, i.e., POI-user, POI-time and

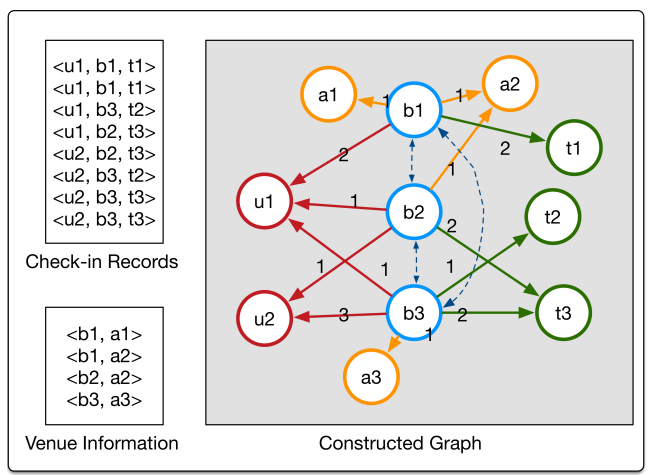

Figure 4: Heterogeneous graph constructed using eight example check-in records and venue information

POI-activity. For POI-time and POI-user edges, edge weights are co-occurrence counts for pair of nodes in the check-in records. For POI-activity edges, edge weights are set to 1 . Our constructed graph contains 6250 user nodes, $221 \mathrm{POI}$ nodes and 7 activities nodes. The POI-user graph density is $22.45 \%$, the POI-time graph density is $82.35 \%$, and the POI-activity graph density is $17.19 \%$.

Note that past work [18] has included POI-POI edges in the graph by considering user transitions from one POI to another. However, these edges increase the average density of our graph substantially (POI-POI density: $60.13 \%$ ). As we will show in the experiments, these edges degrade the performance of the model, particularly on unvisited nodes, so we do not include them in the graph.

\subsection{Graph Embedding}

We adapt the graph embedding approach from Xie et al. [18], which is an extension of [14] geared for POI recommendation. These approaches are all based on the skip-gram model [9] applied to graphs. Given an instance (word/node) and its context (neighbors), the objective of skip-gram is to minimize the log loss of predicting the context using the instance embedding as input features. We employ a similar objective (as described below), but adjust the negative sampling approach to better fit the characteristics of the heterogeneous graph in our setting. 
Specifically, we partition our heterogeneous graph into three bipartite graphs (POI-user graph $G_{b u}$, POI-time graph $G_{b t}$ and POIactivity graph $G_{b a}$ ). Below, we first introduce the graph embedding method for each bipartite graph, then we present our approach for negative sampling, and finally we show how to jointly learn the embeddings over the whole graph.

Bipartite graph embedding. Given a bipartite graph $G_{A B}=$ $\left(V_{A} \cup V_{B}, E\right)$ where $V_{A}$ and $V_{B}$ are two disjoint sets of vertices of different types, and $E$ is the set of edges between them, our task is to find the parameters $\theta$ of a model $p_{\theta}\left(v_{i} \mid v_{j}\right)\left(v_{i} \in V_{A}\right.$ : context vertex; $v_{j} \in V_{B}$ : target vertex) that closely approximates the empirical distribution $\tilde{p}\left(v_{i} \mid v_{j}\right)$ in terms of minimizing cross-entropy. Here the empirical distribution is given by the graph, i.e.,

$$
\tilde{p}\left(v_{i} \mid v_{j}\right)=\frac{w_{i j}}{\operatorname{deg}(j)}
$$

where $w_{i j}$ is the edge weight between $v_{i}$ and $v_{j}$, or zero if $v_{i}$ and $v_{j}$ are not connected.

We define the conditional probability of vertex $v_{i}$ generated by vertex $v_{j}$ as the outcome of a softmax function:

$$
p_{\theta}\left(v_{i} \mid v_{j}\right)=\frac{e^{\vec{z}_{i}^{T} \vec{z}_{j}}}{\sum_{i^{\prime} \in V_{A}} e^{{\overrightarrow{i^{\prime}}}^{T} \vec{z}_{j}}}
$$

where $\overrightarrow{z_{v}}$ denotes the embedding for a vertex $v_{v}$. For each vertex $v_{j}$ in $V_{B}$, Eq.1 defines a conditional distribution $p\left(\cdot \mid v_{j}\right)$ over all the vertices in the set $V_{A}$. For each pair of vertices $v_{j}, v_{j^{\prime}}$, their secondorder proximity can actually be determined by their conditional distributions $p_{\theta}\left(\cdot \mid v_{j}\right), p_{\theta}\left(\cdot \mid v_{j^{\prime}}\right)$.

To learn embeddings that ensure the conditional distribution $p_{\theta}\left(\cdot \mid v_{j}\right)$ closely approximates the empirical distribution $\tilde{p}\left(\cdot \mid v_{j}\right)$, we minimize the following objective function over the graph $G_{A B}$ :

$$
O_{A B}=\sum_{j \in V_{B}} \lambda_{j} d\left(\tilde{p}\left(\cdot \mid v_{j}\right), p_{\theta}\left(\cdot \mid v_{j}\right)\right)
$$

where $d(\cdot, \cdot)$ is the KL-divergence between two distributions, and $\lambda_{j}$ is the importance of vertex $v_{j}$ in the graph. Replacing $d(\cdot, \cdot)$ with KL-divergence, setting $\lambda_{j}=\operatorname{deg}(j)=\sum_{i \in V_{A}} w_{i j}$ and omitting some constants, the objective function can be written as:

$$
O_{A B}=-\sum_{(i, j) \in E} w_{i j} \log p_{\theta}\left(v_{i} \mid v_{j}\right)
$$

Negative sampling. Optimizing the objective in Eq. 3 is computationally expensive, as it requires the summation over the entire set of vertices when calculating the conditional probability $p_{\theta}\left(\cdot \mid v_{j}\right)$. To address this problem, we adopt the approach of negative sampling proposed in word2vec [9], which instead of considering all pairs of nodes, samples a smaller set of observed edges, and then samples multiple "negative" edges for each observed edge. Specifically, in each step, a binary edge $e=(i, j)$ is sampled with the probability proportional to its weight $w_{i j}$, and then multiple negative edges $\left(i^{\prime}, j\right)$ are sampled from a specified noise distribution $q\left(i^{\prime}\right)$.

The default noise distribution used in word2vec (and subsequently used by most, if not all, skip-gram based graph embedding models) is defined as a unigram distribution: $q(i) \propto \operatorname{deg}(i)^{3 / 4}$, where $\operatorname{deg}(i)$ denotes the degree of vertex $v_{i}$. This means that more "popular" vertices are more likely to be selected as negative samples. This makes sense in most NLP and graph embedding problems, where the word co-occurrence matrix or graph adjacency matrix is very sparse. The intuition behind this form of negative sampling is to distinguish between the true context word/vertex and another popular word/vertex which is unlikely to be a context.

However, the graph adjacency matrix is relatively dense in our WiFI check-in data, due to longer user trajectories (i.e., more frequent check-ins). For example, our POI-POI graph adjacency matrix density is $60.13 \%$, whereas in the foursquare dataset the POI-POI graph is extremely sparse with $0.03 \%$ density. If we use the above popularity-based negative sampling method for our data, we find that $96 \%$ of POI vertices sampled as "negatives" are actually connected to the target vertices-which obviously hinders estimation.

To address this issue, we define a new process for efficient negative sampling utilizing the global statistics, i.e., the graph adjacency matrix. Moreover, we integrate the POI categorical information into the noise distribution. When a POI is from a popular category, it's less likely to be a true negative sample, i.e., it's more likely to be connected to the target vertex. By incorporating the global statistics and POI categorical information into the negative sampling procedure, our EDHG model incorporates global features into the local predictive method. In practice, we replace the default noise distribution $q(i)$ with alternative $q(i \mid j)$. Here $v_{j}$ is the given target vertex, and $v_{i}$ is the generated negative sample vertex:

$$
q(i \mid j) \propto 1-\frac{w_{i j}}{\operatorname{deg}(i)} \times \operatorname{Pr}(\operatorname{cat}(i))
$$

where $w_{i j}$ denotes the weight of edge $e_{i j}$, or equals zero if there is no edge between vertex $v_{i}$ and $v_{j}$, and $\operatorname{deg}(i)$ is $v_{i}$ 's degree. $\operatorname{Pr}(\operatorname{cat}(i))$ is the ratio of checkins in POIs with same category as POI $i$, or equals 1 when vertex $i$ is not a POI node. Note that cat $(i)$ corresponds one of the four POI categories in Table 1.

Using edge sampling as in [15] and negative sampling as described above, our final objective function for the bipartite graph $G_{A B}$ is:

$O_{A B}=-\sum_{(i, j) \in E}\left[\log \sigma\left(\vec{z}_{i}^{T} \vec{z}_{j}\right)+\sum_{n=1}^{m} E_{v_{i^{\prime}} \sim q(\cdot \mid j)}\left(\log \sigma\left(-{\overrightarrow{z_{i^{\prime}}}}^{T} \vec{z}_{j}\right)\right)\right]$

Here $\sigma$ refers to the sigmoid function and we sample $m$ negative examples for each positive example. In our implementation, we use the alias table method from Li et al. [7] to draw a negative sample with a pre-computed alias table based on the noise distribution $q(\cdot \mid j)$. This ensures that it takes $\mathrm{O}(1)$ time to repeatedly draw samples from the same distribution. In this way we can achieve the same time complexity as the original LINE model, which is demonstrated to be scalable. Then we adopt the asynchronous stochastic gradient algorithm (ASGD) [12] to optimize Eq. 5. In each iteration, if the edge $e_{i j}$ is sampled, the gradient w.r.t. the embedding vector $\vec{z}_{i}$ of vertex $v_{i}$ will be calculated as $\frac{\partial O_{A B}}{\partial \vec{z}_{i}}$.

Joint training. The overall objective is the sum of the objectives for three bipartite graphs $G_{b u}, G_{b t}$ and $G_{a b}$ :

$$
O=O_{b u}+O_{b t}+O_{a b}
$$

where each component objective $O_{b u}, O_{b t}$ and $O_{a b}$ is specified by Eq. 5 . We learn a joint node embedding by iterating through the three component bipartite graphs in a round-robin fashion and updating the vector representations in each bipartite graph embedding procedure. See Algorithm 1 for more details. 


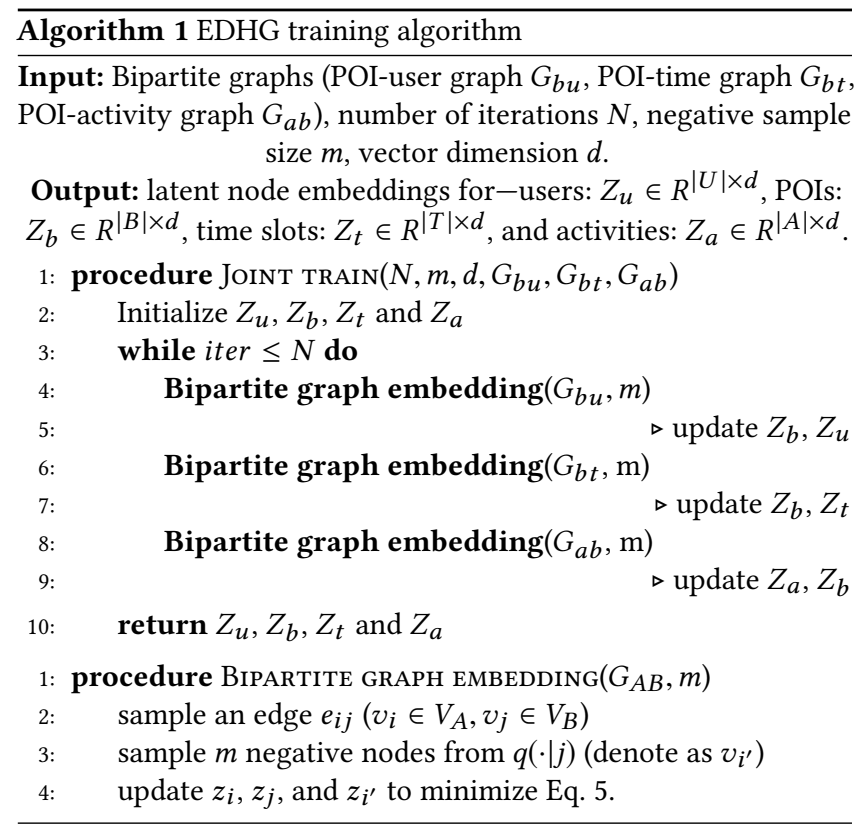

\subsection{Predicting POIs using Embeddings}

Once we have trained our model and learned representations for users, time slots, and locations, we can perform location prediction on new check-in data using simple operations on vectors. Given a query (user, time) i.e., $q=(u, \tau)$, we first project the timestamp $\tau$ into time slot $t$ using the time indexing scheme described in Section 3.1, and then rank the POIs based on their location in the embedding. More precisely, given a query $q=(u, \tau)$, for each POI $b$, we compute its ranking score as:

$$
S(b \mid u, \tau=t)={\overrightarrow{z_{b}}}^{T}{\overrightarrow{z_{u}}}+{\overrightarrow{z_{b}}}^{T} \vec{z}_{t}
$$

where $\overrightarrow{z_{b}}, \overrightarrow{z_{b}}, \overrightarrow{z_{t}}$ are embeddings for user $u$, POI $b$, time slot $t$ respectively. Then we select the $k$ POIs with the highest ranking scores as predictions. Note the POI embedding $\vec{b}$ reflects activity information via the POI-activity graph, since our model jointly learns the embedding of multiple relational networks in the same latent space. Therefore, for both visited POIs and unvisited POIs (also called cold-start POIs), we can perform user recommendations using the same scoring function.

\subsection{Suggesting Friends using Embeddings}

As the embeddings learned from the model fuse the interactions between user-POI, POI-time and POI-activity, we can make use of the embeddings to suggest potential friends for a given user based on their pairwise similarity. Specifically, for a query user $u$, $\forall v \in U \backslash u$, we compute $z_{u}{ }^{T} z_{v}$ and rank the results over $U$, the set of users. From this, we return the top ranked users as people that are more likely to be friends of $u$.

\section{EXPERIMENTAL EVALUATION}

\subsection{Methodology}

In the experiments, we concatenate each student's first $80 \%$ checkin records in chronological order to create the training set examples and then use the remaining $20 \%$ as the test set. We set the number of iterations $(N)$ to $100 \mathrm{M}$ with a batch size of 1 , the dimension of the embedding vector $(d)$ is set to 100 , and we sample 10 negative samples $(m)$ for each vertex pair.

We use accuracy@k as the measure of prediction effectiveness, which is a commonly used metric for this task (see e.g., [4], [18]). However, in contrast with previous work, which only compare the score of the true POI to the score of unvisited POIs during evaluation, we evaluate by comparing the true POI's score to the score of all other POIs (both visited and unvisited). Specifically, for each check-in record (user, time, POI) in the testset, we recommend the top $k$ POIs for the query (user, time) as described in 3.3, and determine if the true POI appears in the top-k list (which is defined as a 'hit'). The accuracy@k is defined as the ratio of hits to the testset size.

\subsection{Comparison Models}

We compare our proposed model EDHG to baselines, state-of-theart alternative methods and EDHG variants.

NBC: Naive Bayes classifier using (user, time-slot) as joint features. For each query $(u, t)$, the probability of predicting POI $b$ is given by $p(b \mid u, t) \propto p(u, t \mid b) \cdot p(b)$ where $b$ denotes a candidate POI, and $(u, t)$ denote a (user, time-slot) pair. This is a strong baseline which takes into account POI popularity and a combination of personal and temporal preference based on counting.

GE [18]: The state-of-art graph embedding method for time-aware POI recommendation (developed using Foursquare and Gowalla data). GE uses POI-POI edges, POI-time edges, POI-region edges, and POI-activity edges, and jointly embeds POIs, times, regions and activities into a latent space. User embeddings are computed as sum of recent visited POIs' embeddings. See Section 5 for details.

GE++: An augmented version of GE that we create to assess the effect of learning user embeddings directly during joint training. This version of GE incorporates POI-user edges in the graph, in addition to its heterogeneous graph embedding.

EDHG: Our proposed model, where we include the POI-user graph, the POI-time graph, and the POI-activity graph with our improved negative sampling method.

EDHG-NS: A simplified version of EDHG, in which we use the traditional method for generating negative samples based on vertex degree.

EDHG-POI: An augmented version of EDHG, where we also include the POI-POI bipartite graph in the heterogeneous graph for learning the embeddings. Note that we only record a POI-POI edge is there is a transition between the two POIs within a four hour time window.

All the models are run on a single machine with $8 \mathrm{G}$ memory using 20 threads. Both EDHG and its variants are very efficient-it 


\begin{tabular}{|c|l|cccc|}
\hline Type & Model & $\mathrm{k}=1$ & $\mathrm{k}=3$ & $\mathrm{k}=5$ & $\mathrm{k}=10$ \\
\hline \multirow{5}{*}{ visited } & GE & 0.1079 & 0.3781 & 0.5104 & 0.6543 \\
& GE++ & 0.3019 & 0.5190 & 0.6063 & 0.6909 \\
& EDHG-NS & 0.3321 & 0.5846 & 0.7024 & 0.8137 \\
& EDHG-POI & 0.6832 & 0.7912 & 0.8368 & 0.8954 \\
& EDHG & $\mathbf{0 . 6 8 4 6}$ & $\mathbf{0 . 7 9 1 5}$ & 0.8367 & 0.8961 \\
& NBC & 0.6765 & 0.7895 & $\mathbf{0 . 8 4 9 5}$ & $\mathbf{0 . 9 0 1 6}$ \\
\hline \multirow{5}{*}{ un- } & GE & 0.0027 & 0.0073 & 0.0241 & 0.0641 \\
& GE++ & $\mathbf{0 . 0 0 8 4}$ & 0.0227 & 0.0332 & 0.0671 \\
& EDHG-NS & 0.0057 & 0.0128 & 0.0301 & 0.0598 \\
& EDHG-POI & 0.0034 & 0.0145 & 0.0195 & 0.0334 \\
& EDHG & 0.0072 & $\mathbf{0 . 0 3 0 7}$ & $\mathbf{0 . 0 3 6 0}$ & $\mathbf{0 . 0 7 1 0}$ \\
& NBC & $5.4 \mathrm{e}-05$ & $6.3 \mathrm{e}-04$ & 0.0025 & 0.0133 \\
\hline \multirow{5}{*}{ total } & GE & 0.1084 & 0.3720 & 0.5026 & 0.6443 \\
& GE++ & 0.2981 & 0.5125 & 0.5988 & 0.6828 \\
& EDHG-NS & 0.3270 & 0.5772 & 0.6937 & 0.7996 \\
& EDHG-POI & 0.6744 & 0.7811 & 0.8261 & 0.8842 \\
& EDHG & $\mathbf{0 . 6 7 6 0}$ & $\mathbf{0 . 7 8 1 6}$ & 0.8263 & 0.8854 \\
& NBC & 0.6677 & 0.7793 & $\mathbf{0 . 8 3 8 5}$ & $\mathbf{0 . 8 9 0 1}$ \\
\hline
\end{tabular}

Table 2: Prediction accuracy

takes about 18 minutes (excluding pre-computation of negative sampling alias table) to process a network with 6486 nodes and 315,407 edges.

\subsection{Predictive Effectiveness}

Here we present the experimental results for all prediction methods using well-tuned parameters. Prediction effectiveness in terms of accuracy@k is shown in Table 2. We report results for visited and unvisited POIs to highlight the difference between in-sample and out-of-sample performance. We also use the $20 \%$ test data to show learning curves for accuracy@1 and @3 in Figures 5, 6 for visited and unvisited POIs respectively. From the results we can make the following observations:

EDHG v.s. EDHG-NS: the full EDHG consistently outperforms EDHG-NS for both visited and unvisited POIs, with a $100 \%$ performance gain in terms of accuracy@1, and 35.4\% in terms of accuracy@3. The significant performance gain is due to the improved negative sampling procedures, which selects more informative negative samples for SGD updates. This indicates that it is promising to customize the empirical noise distribution used in negative sampling for various tasks or datasets.

EDHG v.s. EDHG-POI: The EDHG-POI variant includes the POIPOI transition graph in the original graph which prior work on GE claimed as an important component, but it doesn't improve performance on our recommendation task, and it even downgrades performance for unvisited POIs. This indicates that transition behavior is not informative in our data, as there are too many transitions betweens buildings that cannot be explained by a single reason.

EDHG v.s. GE/GE++: EDHG significantly outperforms GE for both visited and unvisited POIs. The reasons might be due to (1) GE using the POI-POI graph to model the "locality" of individual check-ins for Foursquare data. However, as revealed by the comparison between
EDHG and EDHG-POI, including the POI-POI graph doesn't help in our setting. Or (2) GE doesn't include users as entities in their graph representation, but computes the user embeddings based on recent visit histories. Due to the limited number of POIs in our data, computing the user embedding computed in this way may fail to capture personal preferences. Considering the performance of $\mathrm{GE}++$, which we adapt to our data by adding the POI-user graph to the original GE model, modeling users in the graph helps improve its predictions, but the performance of $\mathrm{GE}++$ is still inferior to that of EDHG.

EDHG v.s. NBC: EDHG achieves comparable performance for visited places and significantly outperforms NBC for unvisited places. In reality, when we look at the learning curves for prediction accuracy, Figure 5 shows that our model converges very fast while NBC needs more data to achieve a comparable result; and Figure 6 shows that our model actually "learns" how to recommend unvisited places with increasing accuracy, while NBC fails to deal with the cold-start recommendation problem, even when provided with a large amount of training data.

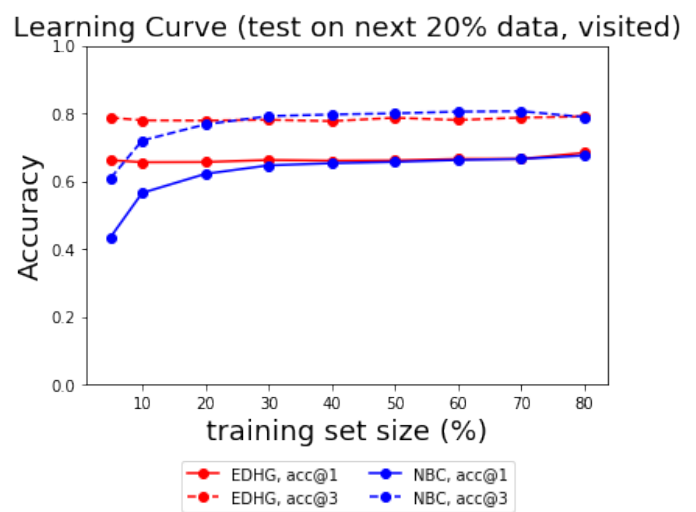

Figure 5: Learning curve for visited POIs

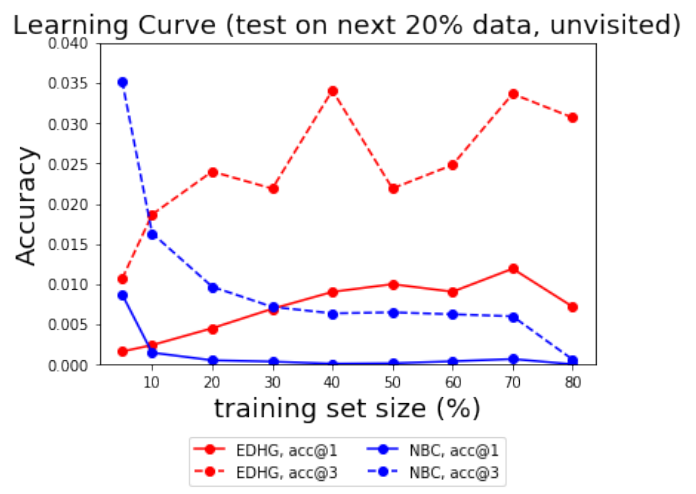

Figure 6: Learning curve for unvisited POIs

\subsection{Parameter Sensitivity}

Granularity of temporal pattern. In Table 2 we evaluated the predictive performance of our model with a combination of weekly of daily pattern using 28 time slots. Here, we design two additional 


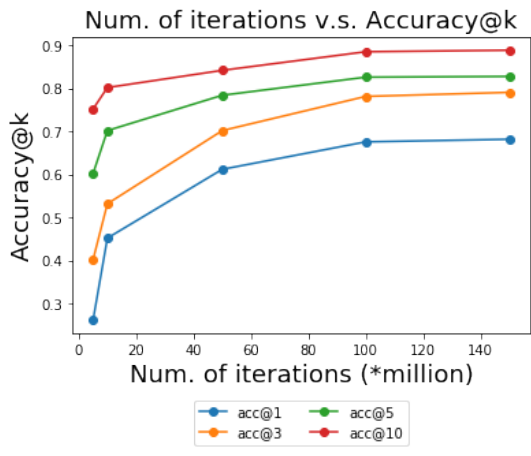

(a) Num. iterations v.s. Prediction accuracy

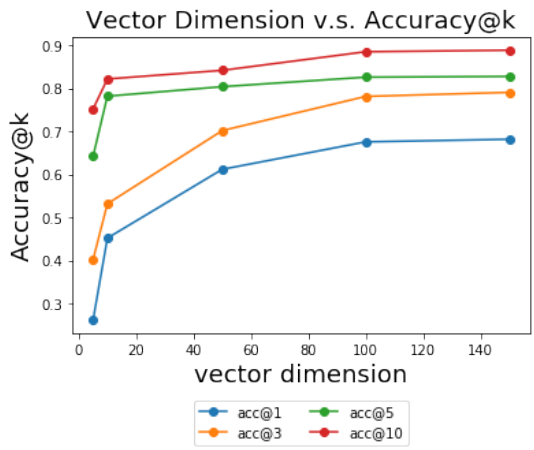

(b) Vector dim. v.s. Prediction accuracy

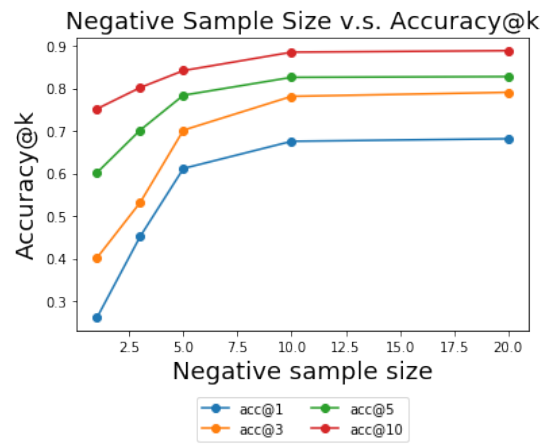

(c) Negative sample size v.s. Prediction accuracy

Figure 7: Impact of number of iterations, embedding vector dimension, and negative sample size on prediction accuracy@k.

\begin{tabular}{|c|l|cccc|}
\hline \multirow{2}{*}{ Type } & Acc@k & $\mathrm{k}=1$ & $\mathrm{k}=3$ & $\mathrm{k}=5$ & $\mathrm{k}=10$ \\
& Model & 0.6025 & 0.7010 & 0.7544 & 0.8403 \\
visited & EDHG-dow & 0.6727 & 0.7850 & 0.8284 & 0.8927 \\
& EDHG-hour & $\mathbf{0 . 6 8 4 6}$ & $\mathbf{0 . 7 9 1 5}$ & $\mathbf{0 . 8 3 6 7}$ & $\mathbf{0 . 8 9 6 1}$ \\
& EDHG & 0.0041 & 0.0083 & 0.0141 & 0.0402 \\
un- & EDHG-dow & 0.0022 & 0.0080 & 0.0260 & 0.0490 \\
visited & EDHG-hour & $\mathbf{0 . 0 0 7 2}$ & $\mathbf{0 . 0 3 0 7}$ & $\mathbf{0 . 0 3 6 0}$ & $\mathbf{0 . 0 7 1 0}$ \\
& EDHG & 0.5947 & 0.6920 & 0.7448 & 0.8299 \\
& EDHG-dow & 0.6639 & 0.7727 & 0.8102 & 0.8821 \\
total & EDHG-hour & $\mathbf{0 . 6 7 6 0}$ & $\mathbf{0 . 7 8 1 6}$ & $\mathbf{0 . 8 2 6 3}$ & $\mathbf{0 . 8 8 5 4}$ \\
& EDHG & &
\end{tabular}

Table 3: Prediction accuracy v.s. temporal granularity.

variants to explore the effect of temporal patterns with different granularity. The EDHG-hour only considers time period of day (4 time slots) and EDHG-dow only considers day of week (7 time slots). The results are shown in Table 3.

From Table 3 we can see that both Day of Week and Hour of Day are important temporal factors. Specifically, when we only consider weekly patterns (day of week), prediction accuracy decreases by roughly $10 \%$; when we only consider daily patterns (hour of day), prediction accuracy slightly decreases by $1.5 \%$. This indicates that time-of-day effects are more significant than day-of-week effects in terms of POI prediction. It's likely that students, particularly freshman, have less flexibility in daily routines due to their course schedule, which makes time-of-day a more important factor for nearly all types of activities.

Number of iterations \& vector dimension. Figures $7 \mathrm{a}$ and $7 \mathrm{~b}$ show the performance of EDHG with different number of iterations $N$ and embedding dimensions $d$. Note that, the units for $N$ is set to 1 million. We can see from Figure 7a that the accuracy increases and converges quickly when the number of iterations is larger than $50 \mathrm{M}$. We used $N=100(M)$ to ensure convergence. For embedding dimension, we chose $d=100$ as the accuracy does not increase substantially after that point.

Negative sample size. Figure $7 \mathrm{c}$ presents the performance of EDHG with different numbers of negative samples per example. With more negative samples the accuracy increases, and it plateaus when negative sample size is 10 . Therefore, we chose $m=10$ negative samples for use during optimization.

\subsection{Friend Suggestion Effectiveness}

To examine the efficacy of using EDHG's vector representation for suggesting friends, we first need to identify a proxy signal for evaluation (since we do not have ground truth information about friend relations among the students). Specifically, we consider the following two ways data to determine "true" friends for evaluation:

Covisit As in shown in Section 2.3, a co-visitation record is generated when two users check in at the same building at the same time (time unit: minute). In this approach, we identify "friends" of a query user as those with the largest co-visitation counts.

Location Based on the user-building check-in count matrix, we create a ranking list of buildings for each user, with the most frequently visited building ranked highest. In this approach, we identify "friends" of a query user as those that have the smallest distance between the users' ranked list of buildings (using Kendall $\tau$ distance). We apply the friend suggestion to the most active users in our dataset, sorted by activity level. For each user, given a set of "true" friends from one of the baselines above, we evaluate the top 10 friend suggestions from EDHG using Mean Reciprocal Rank (MRR). MRR is computed as:

$$
\frac{1}{|U|} \sum_{i=1}^{|U|} \sum_{j \in F_{i}} \frac{1}{\operatorname{rank}(j)}
$$

where $U$ is the set of active users, $F_{i}=10$ is the set of "true" friends of user $i$ which are obtained from the data and $\operatorname{rank}(j)$ is the rank of item $j$ in the ranking list. We compare the performance of EDHG and GE in terms of MRR scores.

Since the Covisit baseline encodes both temporal and geographical preference, and the Location baseline only takes into account geographical preference, Covisit is likely a better proxy for "true" friends, and thus the ideal search results should have a higher MRR score w.r.t. Covisit.

The results are shown in Figure 8. We can make the following observations based on the results. Comparing Covisit and Location, EDHG suggests friends with more relevance to co-visitation 
counts than merely geographical preference, while GE does the opposite. Since neither method uses user co-visitation data directly in its model, this implies that EDHG captures social behaviors from the spatial-temporal data more accurately. Comparing the general MRR scores of the two models, EDHG suggests friends with higher accuracy in general. We also calculated the MMR between the Covisit and Location friend lists (the green line in the graph). The relatively low MRR score reveals that a large potion of co-visitation behavior cannot be explained by location preference. In reality, students with same major and same year usually stay in a same set of places, e.g. academic buildings and libraries, but their temporal preferences may vary significantly. The plot shows how performance changes as the increase of $|U|$. We can see that, on the co-visitation data, EDHG's MMR score decreases as less active users are included in $U$. This indicates that EDHG discovers better suggestions for more active users, which suggest that with richer check-in information we can capture more precise social relationships.

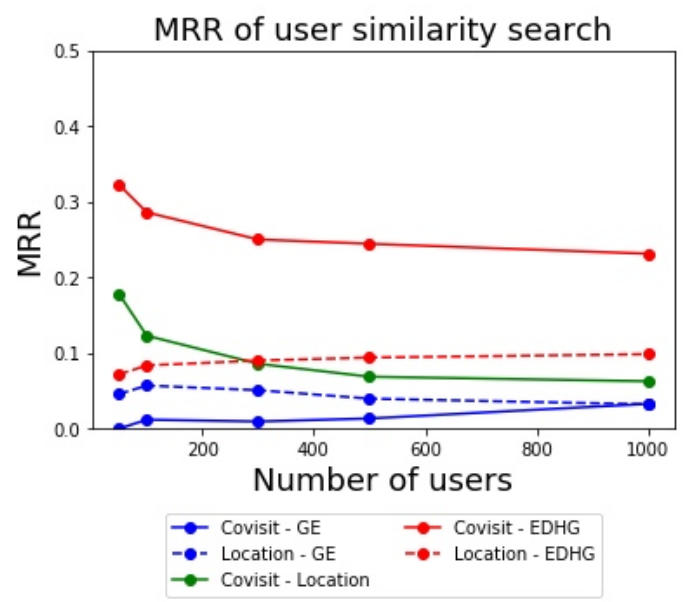

Figure 8: Number of frequent users v.s. MRR scores

\subsection{Visualization of Embeddings}

Figure 9 shows a visualization of the learned user embeddings, where we project the $d=100$ dimensions into 2D using t-SNE [8]. From the visualization we can clearly find two clusters of computer science students and pharmacy students (colored green and red respectively). This can be understood through their differences in temporal preference (as shown in Figures 1c-1d) and also their geographical preferences (i.e., these two majors share very few academic buildings).

\section{RELATED WORK}

POI recommendation methods have received extensive research attention in the last five years, and many approaches have been proposed. For example, Wang et al. $([16,17])$ applied sparse additive generative models to incorporate multiple factors for POI recommendation. Yin et al. [21] proposed a Spatial-Aware Hierarchical Collaborative Deep Learning model (SH-CDL), which jointly performs deep representation learning for POIs and hierarchically

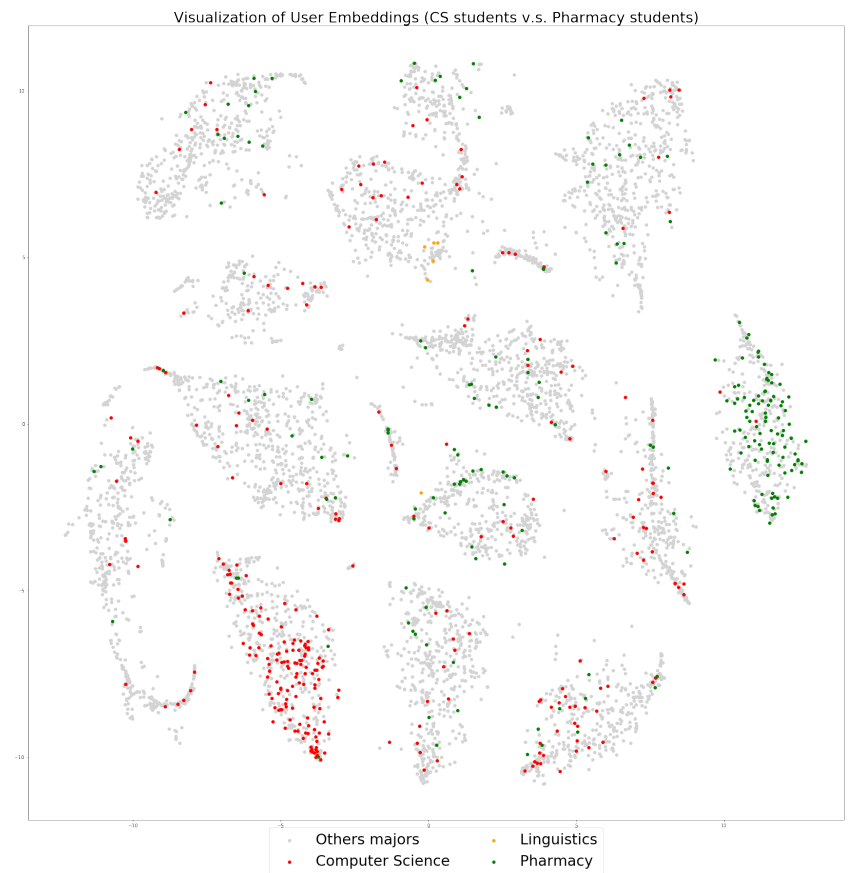

Figure 9: User embeddings

additive representation learning for spatial-aware personal preferences. Xie et al. [18] proposed a graph embedding model GE for context-aware POI recommendation, which uses the POI-POI transition graph, POI-region graph, and POI-category graph, and jointly learn the representations for POI, region, and time with the same method as PTE [14]. User embeddings are then computed as weighted sum of recent POI embeddings, and with user, POI, time embeddings they can perform time-aware POI recommendation. GE achieved better performance than all previous work on this task, which is why we use it as a baseline for evaluation in this paper.

In addition, our work is related to the extensive literature on network embedding, which has attracted a great deal of attention in recent years. Many of these recent methods are technically inspired by Skipgram [9]. For example, Deepwalk [11] uses the embedding of a node to predict the context in the graph, where the context is generated by a random walk. Metapath2vec [3] extends DeepWalk for heterogeneous graph embedding. LINE [15] extends the skipgram model to have multiple context spaces for modeling both first and second order proximity. PTE [14] adapts the LINE model [15] for embedding bipartite networks. Note that PTE model is directly adopted by GE [18] for POI recommendation, and we further adjust PTE to our setting by improving the negative sampling method and targeting the graph construction process.

Heterogeneous information network embedding has been broadly applied to multiple tasks. For example, Tang et al. [14] predicted text embeddings based on heterogeneous text networks which showed great potential in document classification. Zhang et al. [22] proposed ReAct, a method that processes continuous geo-tagged social media (GTSM) streams into a heterogeneous graph and obtains recency-aware activity models on the fly, in order to reveal up-to-date spatiotemporal activities. Chen and Sun [2] proposed a 
task-guided and path-augmented heterogeneous network embedding for author identification task.

\section{CONCLUSIONS}

This paper presents our analysis of the first educational "check-in" dataset and proposes EDHG, a heterogeneous graph embedding based method to model more dense spatio-temporal checkin activity. To account for the unique characteristics of the data, we improve the negative sampling method to incorporate global statistics of the graph/data into the noise distribution. We also show that it's better to drop the POI-POI transition edges when the check-in data is more dense. We evaluated EDHG with two tasks: time-aware POI prediction and friend suggestion. On both tasks, our proposed model outperforms the previous state-of-art methods and baselines. These initial results indicate the promise of using student trajectory information for personalized recommendations in education apps, as well as in predictive models of student retention and satisfaction.

Several interesting research problems remain for further exploration. For example, we did not make direct use of the co-visitation data in the model but rather withheld it for evaluation of the friend suggestions. We plan to incorporate it in the training process and see whether social interactions impact student checkin behavior. Also, inspired by Chen et al. [1], we may be able to further improve the negative sampling by dynamically selecting informative negative samples during each SGD update.

\section{ACKNOWLEDGEMENTS}

This research is supported by NSF under contract number(s) IIS1546488 and IIS-1618690. The U.S. Government is authorized to reproduce and distribute reprints for governmental purposes notwithstanding any copyright notation hereon. The views and conclusions contained herein are those of the authors and should not be interpreted as necessarily representing the official policies or endorsements either expressed or implied, of NSF or the U.S. Government.

\section{REFERENCES}

[1] Long Chen, Fajie Yuan, Joemon M Jose, and Weinan Zhang. 2018. Improving Negative Sampling for Word Representation using Self-embedded Features. In Proceedings of the Eleventh ACM International Conference on Web Search and Data Mining. ACM, 99-107.

[2] Ting Chen and Yizhou Sun. 2017. Task-guided and path-augmented heterogeneous network embedding for author identification. In Proceedings of the Tenth ACM International Conference on Web Search and Data Mining. ACM, 295-304.

[3] Yuxiao Dong, Nitesh V Chawla, and Ananthram Swami. 2017. metapath2vec: Scalable representation learning for heterogeneous networks. In Proceedings of the 23rd ACM SIGKDD International Conference on Knowledge Discovery and Data Mining. ACM, 135-144.

[4] Shanshan Feng, Xutao Li, Yifeng Zeng, Gao Cong, Yeow Meng Chee, and Quan Yuan. 2015. Personalized Ranking Metric Embedding for Next New POI Recommendation.. In IFCAI. 2069-2075.

[5] Jing He, Xin Li, Lejian Liao, Dandan Song, and William K Cheung. 2016. Inferring a Personalized Next Point-of-Interest Recommendation Model with Latent Behavior Patterns.. In AAAI. 137-143.

[6] S Kylasa, G Kollias, and A Grama. 2016. Social ties and checkin sites: connections and latent structures in location-based social networks. Social Network Analysis and Mining 6, 1 (2016)

[7] Aaron Q Li, Amr Ahmed, Sujith Ravi, and Alexander J Smola. 2014. Reducing the sampling complexity of topic models. In Proceedings of the 20th ACM SIGKDD international conference on Knowledge discovery and data mining. ACM, 891-900.

[8] L Maaten and G Hinton. 2008. Visualizing data using t-SNE. Fournal of machine learning research 9, Nov (2008).

[9] Tomas Mikolov, Ilya Sutskever, Kai Chen, Greg S Corrado, and Jeff Dean. 2013. Distributed representations of words and phrases and their compositionality. In Advances in neural information processing systems. 3111-3119.
[10] Anastasios Noulas, Salvatore Scellato, Cecilia Mascolo, and Massimiliano Pontil. 2011. An empirical study of geographic user activity patterns in foursquare. ICWSM 11, 70-573 (2011), 2.

[11] Bryan Perozzi, Rami Al-Rfou, and Steven Skiena. 2014. Deepwalk: Online learning of social representations. In Proceedings of the 20th ACM SIGKDD international conference on Knowledge discovery and data mining. ACM, 701-710.

[12] Benjamin Recht, Christopher Re, Stephen Wright, and Feng Niu. 2011. Hogwild: A lock-free approach to parallelizing stochastic gradient descent. In Advances in neural information processing systems. 693-701.

[13] Yizhou Sun, Hongzhi Yin, and Xiang Ren. 2017. Recommendation in context-rich environment: An information network analysis approach. In Proceedings of the 26th International Conference on World Wide Web Companion. International World Wide Web Conferences Steering Committee, 941-945.

[14] Jian Tang, Meng Qu, and Qiaozhu Mei. 2015. Pte: Predictive text embedding through large-scale heterogeneous text networks. In Proceedings of the 21th ACM SIGKDD International Conference on Knowledge Discovery and Data Mining. ACM, $1165-1174$.

[15] Jian Tang, Meng Qu, Mingzhe Wang, Ming Zhang, Jun Yan, and Qiaozhu Mei. 2015. Line: Large-scale information network embedding. In Proceedings of the 24th International Conference on World Wide Web. International World Wide Web Conferences Steering Committee, 1067-1077.

[16] Weiqing Wang, Hongzhi Yin, Ling Chen, Yizhou Sun, Shazia Sadiq, and Xiaofang Zhou. 2015. Geo-SAGE: A geographical sparse additive generative model for spatial item recommendation. In Proceedings of the 21th ACM SIGKDD International Conference on Knowledge Discovery and Data Mining. ACM, 1255-1264.

[17] Weiqing Wang, Hongzhi Yin, Shazia Sadiq, Ling Chen, Min Xie, and Xiaofang Zhou. 2016. Spore: A sequential personalized spatial item recommender system. In Data Engineering (ICDE), 2016 IEEE 32nd International Conference on. IEEE, 954-965.

[18] Min Xie, Hongzhi Yin, Hao Wang, Fanjiang Xu, Weitong Chen, and Sen Wang. 2016. Learning graph-based poi embedding for location-based recommendation. In Proceedings of the 25th ACM International on Conference on Information and Knowledge Management. ACM, 15-24.

[19] Carl Yang, Lanxiao Bai, Chao Zhang, Quan Yuan, and Jiawei Han. 2017. Bridging Collaborative Filtering and Semi-Supervised Learning: A Neural Approach for POI Recommendation. In Proceedings of the 23rd ACM SIGKDD International Conference on Knowledge Discovery and Data Mining. ACM, 1245-1254.

[20] Zijun Yao. 2018. Exploiting Human Mobility Patterns for Point-of-Interest Recommendation. In Proceedings of the Eleventh ACM International Conference on Web Search and Data Mining. ACM, 757-758.

[21] H Yin, W Wang, H Wang, L Chen, and X Zhou. 2017. Spatial-Aware Hierarchical Collaborative Deep Learning for POI Recommendation. IEEE Transactions on Knowledge and Data Engineering 29, 11 (2017).

[22] Chao Zhang, Keyang Zhang, Quan Yuan, Fangbo Tao, Luming Zhang, Tim Hanratty, and Jiawei Han. 2017. ReAct: Online Multimodal Embedding for RecencyAware Spatiotemporal Activity Modeling. In Proceedings of the 40th International ACM SIGIR Conference on Research and Development in Information Retrieval. ACM, 245-254.

[23] Yu Zheng, Lizhu Zhang, Xing Xie, and Wei-Ying Ma. 2009. Mining interesting locations and travel sequences from GPS trajectories. In Proceedings of the 18th international conference on World wide web. ACM, 791-800. 